\title{
BMJ Open Efficacy of biofeedback, repetitive transcranial magnetic stimulation and pelvic floor muscle training for female neurogenic bladder dysfunction after spinal cord injury: a study protocol for a randomised controlled trial
}

\author{
Lin Xu (D) , ${ }^{1,2}$ Chenying Fu, ${ }^{3}$ Qing Zhang, ${ }^{1,2}$ Feng Xiong, ${ }^{1,2}$ Lihong Peng, ${ }^{1,2}$
} Zejun Liang, ${ }^{1,2}$ Li Chen, ${ }^{1,2}$ Chengqi He, ${ }^{1,2}$ Quan Wei ${ }^{1,2}$

To cite: Xu L, Fu C, Zhang Q, et al. Efficacy of biofeedback, repetitive transcranial magnetic stimulation and pelvic floor muscle training for female neurogenic bladder dysfunction after spinal cord injury: a study protocol for a randomised controlled trial. BMJ Open 2020;10:e034582. doi:10.1136/ bmjopen-2019-034582

- Prepublication history and additional material for this paper are available online. To view these files, please visit the journal online (http://dx.doi. org/10.1136/bmjopen-2019034582).

LX and CF contributed equally.

Received 29 November 2019 Revised 14 May 2020 Accepted 23 June 2020

Check for updates

(c) Author(s) (or their employer(s)) 2020. Re-use permitted under CC BY-NC. No commercial re-use. See rights and permissions. Published by BMJ.

For numbered affiliations see end of article.

Correspondence to Professor Quan Wei; weiquan@scu.edu.cn

\section{ABSTRACT}

Introduction Neurogenic bladder dysfunction is prevalent in female patients with spinal cord injury (SCI), and previous guidelines have recommended pelvic floor muscle training (PFMT) for first-line conservative treatment. However, the actual regimen of PFMT varies widely and the single treatment does not satisfy the need of some patients. Therefore, this study aims to provide a detailed rationale and methodology for comparing the effectiveness of PFMT, biofeedback and repetitive transcranial magnetic stimulation (rTMS) as adjunct treatments for neurogenic bladder dysfunction.

Methods and analysis This trial is a single-centre randomised controlled trial for female patients with urinary incontinence (UI) in phase of chronic SCl. Eligible participants will be randomised to one of four arms: (1) PFMT, (2) PFMT with biofeedback, (3) PFMT and rTMS and (4) PFMT with biofeedback and rTMS. There will be 44 participants in each arm and all the subjects will undergo 20 treatment sessions, five times a week for 4 weeks. The outcomes will be evaluated at 4 weeks, 3 months and 6 months after randomisation. The primary outcome is the International Consultation on Incontinence Questionnaire-Urinary Incontinence Short Form, and the secondary outcomes include bladder diary, pelvic floor muscle function and the International Spinal Cord Injury Quality of Life Basic Data Set. Ethics and dissemination The Clinical Research and Biomedical Ethics Committee of the West China Hospital, Sichuan University has approved this trial and the approval number is 2019-885. All participants will be provided written informed consent after verification of the eligibility criteria. The results of this study will be accessible in peer-reviewed publications and be presented at academic conferences. Trial registration number Chinese Clinical Trial Registry (ChiCTR1900026126).

\section{INTRODUCTION}

The prevalence of urinary incontinence (UI) is notable in spinal cord injury (SCI) populations, affecting over half $(52.3 \%)$
Strengths and limitations of this study

- This is the first randomised trial in China examining the effectiveness and safety of pelvic floor muscle training, biofeedback and repetitive transcranial magnetic stimulation as therapies for neurogenic bladder dysfunction.

- Both subjective and objective tools to assess bladder function and quality of life are used in this study.

- All study participants are required to attend standardised training.

- A sham comparator will not be used in these trials, so the participant blinding and practitioner blinding cannot be achieved, and complete assessor blinding may be challenging because of the self-reported outcome measurement.

- Participants might be heterogeneous because of the broad age range, different levels of spinal cord injury and types of neurogenic bladder dysfunction.

of the patients. ${ }^{1}$ A considerable number of patients with SCI are dissatisfied with their voiding function and ask for specific management. $^{2} 3$ Neurogenic bladder dysfunction brought by SCI is an area of great interest for several decades, and it can cause a significant socioeconomic burden. In previous studies, patients with SCI experienced urogenital diseases, motor, sensory and autonomic function impairment and poor quality of life (QoL). ${ }^{4-6}$ Notably, the type of neurogenic bladder dysfunction is significantly associated with the degree of injury in patients with SCI. Lesions located in the spinal cord between the sacrum and the brainstem mostly lead to neurogenic detrusor overactivity (NDO) and detrusor-sphincter dyssynergia. The patients with damage to the sacral part of the spinal 
cord usually show detrusor areflexia, resulting in urinary retention and/or incontinence owing to urine overflow or a loss of urethral resistance. ${ }^{7}$ Clinicians and researchers must give an individually tailored management strategy based on a specific diagnosis in each different individual.

Pelvic floor muscle training (PFMT) is a wellestablished first-line conservative treatment of UI, five times more likely to report symptomatic cure than no treatment or inactive control treatments. ${ }^{8}$ The Dutch College of General Practitioners' (NHG) practice guideline suggested exercise therapy could be employed for women with UI under supervision of a pelvic physical therapist. ${ }^{9}$ To date, many studies have confirmed that PFMT is safe and effective in treating bladder dysfunction and improving QoL. ${ }^{10} 11$ The mechanism of PFMT is increasing urethral pressure, supporting of the bladder neck, and interacting with the transversus abdominis via coordinated contractions between the pelvic floor muscles and the transversus abdominis, improving bladder function finally. ${ }^{12}{ }^{13}$ While numerous guidelines recommend PFMT, the actual regimen of PFMT in clinical settings varies widely. There is insufficient evidence to get a standardised PFMT regimen.

Biofeedback has been used, as a common adjunct to PFMT, for women with UI in the past years. Almost all researchers have thought biofeedback can add benefit to PFMT in theory. Biofeedback is defined as visual feedback and frequent verbal feedback provided by the computer, helping participants to observe muscle contraction and relaxation. In previous studies, ${ }^{14} 15$ clinicians and physical therapists could master readaptation situations of patients in the setting of pelvic dysfunction by evaluating the myoelectric activation of these muscle groups in biofeedback. However, most reviews published in succession showed that there was not a significant difference in outcomes comparing PFMT with biofeedback and PFMT, which might be caused by low-quality randomised controlled trials (RCT). ${ }^{1617}$

More and more scientific articles have reported that electrical stimulation is second-line or third-line treatment for neurogenic lower urinary tract disease, and this conclusion is now well-validated and accepted. ${ }^{18}$ Some articles reported that repetitive transcranial magnetic stimulation (rTMS) had been extensively used in pain management by affecting nuclei in the thalamus and subthalamic regions. ${ }^{19} 20$ In a recent review, Ellaway et al purported the applications of rTMS not only relieving neuropathic pain, but also addressing motor control in SCI. ${ }^{21}$ Despite the potential treatment effects of rTMS to be used in the restoration of bladder and bowel sphincter function, the use of rTMS was reported to be uncommon in clinical settings and there was considerable variation in clinical outcomes. ${ }^{21} 22$

In Chinese medical clinical settings, PFMT has been frequently used for treating UI. Biofeedback and rTMS, as potential adjunct treatments, are occasionally used in patients with incomplete SCI who retain some feeling or motor function below the site of the injury and are evaluated as B, C or D level according to the American Spinal Injury Association Impairment Scale. However, there is lack of research evidence to get consistent conclusions for the clinical effectiveness of these interventions. To our knowledge, no clinical research has been conducted to explore the effect of combing PFMT and rTMS. Thus, the primary aim of this study is to investigate the effectiveness and safety of PFMT, biofeedback and rTMS as adjunct treatments for neurogenic bladder dysfunction. Our secondary objective is to explore a standardised regimen which consists of PFMT and other conservative treatment protocol for women with neurogenic bladder dysfunction.

\section{METHODS AND ANALYSIS \\ Study design}

This trial is a single-centre randomised controlled trial with a follow-up period of 6 months. All subjects will receive observations and baseline assessments after obtaining informed consent (online supplementary additional file 1), only those who meet inclusion/ exclusion criteria will be included in the trial and randomised. The subjects will be randomly allocated to either arm 1,2, 3 or 4 in a 1:1:1:1 ratio by randomisation sequence (table 1 ), including (1) PFMT, (2) PFMT with biofeedback, (3) PFMT and rTMS and (4) PFMT with biofeedback and rTMS. ${ }^{23}$ This randomisation process will use a computer-generated randomisation sequence, an outsider research-assistant will allocate these numbers to participants according to the order of inclusion. In this trial, participant blinding and practitioner blinding cannot be achieved because they will be aware of the intervention methods and personally take part in treatments. The primary investigator who will assess the outcomes and analyse data will be blinded to the group allocation by not involving in the treatment administration. The participant flow for this trail is presented in figure 1. The protocol follows the Standard Protocol Items: Recommendations for Interventional Trials (SPIRIT) guidelines and fulfils the SPIRIT checklist $^{24}$ (online supplementary additional file 2 ).

\section{Participants}

Over the trial recruitment period, investigators will recruit potentially eligible women. In the hospital, clinical experts will confirm a clinical diagnosis of neurogenic bladder dysfunction after SCI, and assess other trial eligibility criteria. If a woman is eligible, the researchers will ask about her wish and obtain informed consent. Box 1 lists the inclusion and exclusion criteria. ${ }^{25}$

Participants fulfilling the above criteria will receive further assessments, including bladder diary, pelvic floor muscle function, International Consultation on Incontinence Questionnaire-Urinary Incontinence Short Form (ICIQ-UI SF) ${ }^{26}$ and the International Spinal Cord Injury Quality of Life Basic Data Set (SCI-QoL). ${ }^{27}$ This trial will happen in Southwest China, when all participants are referred to the Neurological Rehabilitation Division, 
Table 1 Standardisation of training and protocols

\begin{tabular}{|c|c|c|c|}
\hline Group & $\begin{array}{l}\text { Standardised } \\
\text { training } \\
\text { programme }\end{array}$ & Dosage & $\begin{array}{l}\text { Time in } \\
\text { total }\end{array}$ \\
\hline \multirow[t]{3}{*}{ PFMT } & $\begin{array}{l}\text { Sustained } \\
\text { contractions }\end{array}$ & $\begin{array}{l}\text { The patients will complete pelvic muscle contraction lasting } 6-10 \text { s per time, then } \\
\text { start next contraction after resting for } 10 \mathrm{~s}, 8-10 \text { repetitions per set, 1-2 sets per } \\
\text { session. }\end{array}$ & \multirow[t]{3}{*}{$30 \mathrm{~min}$} \\
\hline & $\begin{array}{l}\text { Phasic } \\
\text { contractions }\end{array}$ & $\begin{array}{l}\text { The patients will complete pelvic muscle contraction lasting } 2-5 \text { s per time, then } \\
\text { rest, the resting time is twice as long as the contraction time, } 10 \text { repetitions per } \\
\text { set, } 1-3 \text { sets per session. }\end{array}$ & \\
\hline & $\begin{array}{l}\text { Guide-condition } \\
\text { training }\end{array}$ & $\begin{array}{l}\text { The patients will be asked to simulate the activity like cough, sneeze while } \\
\text { contracting the pelvic floor muscles, adapting to the needs of daily life. }\end{array}$ & \\
\hline \multirow{2}{*}{$\begin{array}{l}\text { PFMT with } \\
\text { biofeedback }\end{array}$} & PFMT protocol & Exercise prescription is the same with PFMT group. & \multirow[t]{2}{*}{$30 \mathrm{~min}$} \\
\hline & $\begin{array}{l}\text { Biofeedback } \\
\text { protocol }\end{array}$ & $\begin{array}{l}\text { Participants will receive visual or auditory feedback from biofeedback equipment } \\
\text { to monitor and correct pelvic floor muscle contractions during training. }\end{array}$ & \\
\hline \multirow{3}{*}{$\begin{array}{l}\text { PFMT with } \\
\text { biofeedback } \\
\text { and rTMS }\end{array}$} & PFMT protocol & Exercise prescription is the same with PFMT group & \multirow[t]{3}{*}{$50 \mathrm{~min}$} \\
\hline & $\begin{array}{l}\text { Biofeedback } \\
\text { protocol }\end{array}$ & $\begin{array}{l}\text { Participants will receive visual or auditory feedback from biofeedback equipment } \\
\text { to monitor and correct pelvic floor muscle contractions during training. }\end{array}$ & \\
\hline & rTMS protocol & $\begin{array}{l}\text { The patients will receive rTMS treatment at } 5 \mathrm{~Hz} \text { for } 20 \mathrm{~min} \text { after determining the } \\
\text { hemisphere and specific part to stimulate. }\end{array}$ & \\
\hline
\end{tabular}

PFMT, pelvic floor muscle training; rTMS, repetitive transcranial magnetic stimulation.

they will receive center-intervention, which is centerbased rehabilitation under supervision of therapists in the Department of Rehabilitation Medicine Center. Different groups will receive corresponding interventions according to plans, and all equipment needed to be used will be provided by the Department of Rehabilitation Medicine Center.

\section{Interventions}

All subjects in this trial will receive $\mathrm{PFMT}^{28}$ because level 1 evidence indicates that PFMT is effective in treating women with pelvic floor muscle dysfunctions, such as stress, urgency or mixed UI. ${ }^{28}$ Vásquez et al summarised two case studies and reported that a 6 -week programme of PFMT might have a beneficial effect on promoting voluntary control of NDO and might reduce incontinence in selected cases with a motor incomplete spinal cord lesion. ${ }^{29}$ Overall PFMT effectiveness depends on exercise adherence largely. The trial will increase continued adherence by two methods, first, patients will receive rehabilitation directly after referred from other departments to the rehabilitation department. Besides that, the patients will complete the bladder diaries during the treatment to urge them into training.

\section{Control group (PFMT)}

After randomisation, trained physiotherapists will teach subjects the correct exercise techniques and correct contraction methods, and will encourage participants to feel the contraction and relaxation of muscles by palpation when they are training in the supine position. The individual subject and her physiotherapist will identify the initial PFMT programme together, the exercise programme will be practiced during hospitalisation and is allowed to adjust by the therapist according to the patient's condition. Women in the PFMT group will receive intervention during first 4 weeks, and will be followed up for 6 months. PFMT protocol obeys exercise prescription principles, including frequency, intensity, time, type, volume and progress. This study determines initial prescription based on the PERFECT (Power, Endurance, Repetitions, Fast, Every Contraction Timed) assessment. ${ }^{30}$ A 30 min session is divided into three parts, five times weekly for a total of 20 sessions, part 1 is sustained contractions lasting $6-10 \mathrm{~s}(\mathrm{~s})$, with the resting of $10 \mathrm{~s}, 8-10$ repetitions, $1-2$ sets; part 2 is phasic contractions lasting $2-5 \mathrm{~s}$, with twice the resting time, 10 repetitions, 1-3 sets; part 3 is guide-condition training, asking the patient to simulate the activity of increased intra-abdominal pressure (such as cough, sneeze) while contracting the pelvic floor muscles. ${ }^{31}$ The intensity will be set according to the maximum duration of pelvic floor muscle contraction when this patient feels tired. The programme will be progressed when the woman's ability is reaching her individualised goal. For example, if a patient can only complete lasting $6 \mathrm{~s}$ muscle contraction per time due to bad body condition, her prescription will be pelvic muscle contraction lasting $6 \mathrm{~s}$ per time, then resting for $10 \mathrm{~s}, 8$ repetitions per set, 1 set per session. 


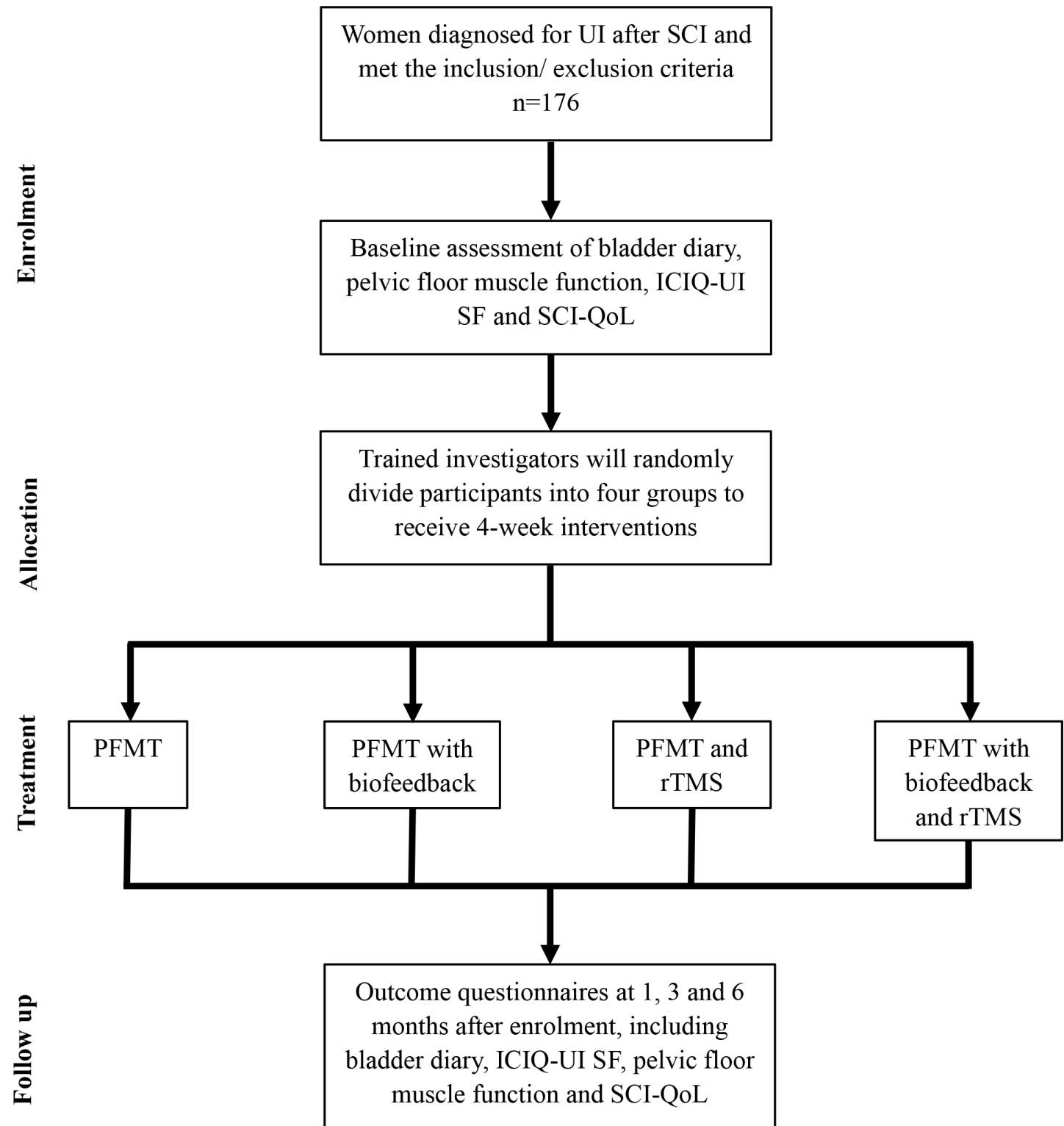

Figure 1 Proposed participant flow. ICIQ-UI SF, International Consultation on Incontinence Questionnaire-Urinary Incontinence Short Form; PFMT, pelvic floor muscle training; rTMS, repetitive transcranial magnetic stimulation; SCI, spinal cord injury; SClQoL, International Spinal Cord Injury Quality of Life Basic Data Set; UI, urinary incontinence.

With the improvement of the patient's physical condition, the duration of contraction, the number of repetitions and sets in the prescription will gradually increase to meet the principles of exercise physiology.

There is not any assistant equipment that can help patients to complete muscle training (eg, biofeedback, rTMS) used in the control group but patients' verbal feedback and palpated feedback is permitted.

\section{Group 1 (PFMT with biofeedback)}

Biofeedback is a mind-body technique to gain control over involuntary bodily functions, and it is a behavioural learning process that relies on continuous operation and correction. This process involves being connected to a device with sensors that provide feedback about specific aspects of participants' bodies. Participants who are randomised to receiving PFMT with biofeedback will complete the same PFMT protocol as the control group, in addition, five electrodes will be placed in the vaginal area manually by the physiotherapist to connect biofeedback device. Participants receive visual, auditory, or verbal feedback from equipment to correct their reactions. ${ }^{14}$ All participants will complete whole training programmes under the commands of a computer after understanding the training protocol that would be performed. The participants will use the same type of device throughout the trial. ${ }^{15}$

\section{Group 2 (PFMT and rTMS)}

Studies have demonstrated that extensive reorganisation occurs in human's corticospinal tract, primary motor cortex (M1) and spinal cord after SCI. ${ }^{32}$ Moreover, the researchers who explore the effect of rTMS have found that it can elicit long-lasting changes in the excitability of 


\section{Box 1 Inclusion and exclusion criteria of the study}

\section{Inclusion criteria}

1. Patients who are women aged $18-75$ years with incomplete $\mathrm{SCl}$.

2. Patients who are in phase of chronic $\mathrm{SCl}$ without the risk of death.

3. Patients who have UI with a total score of $\geq 8$ on the ICIQ-UI SF.

4. Patients who are willing and suitable to be randomised.

\section{Exclusion criteria}

1. Patients who are motor completeness of injury (A or B on the American Spinal Injury Association Impairment Scale).

2. Patients who have cognitive problems including uncontrolled epilepsy, central nervous system diseases or psychosis, and cannot cooperate with rehabilitation training.

3. Patients who have metal in cranium, intracardiac lines, increased intracranial pressure, heart disease, cardiac pacemaker, medication pump, tricyclic antidepressants, neuroleptics or family history of epilepsy so that they cannot receive rTMS treatment.

4. Patients who are pregnant or are less than 6 months postnatal.

5. Patients who have had formal instruction in PFMT in last years.

6. Patients who are currently participating in another research relating to their UI.

ICIQ-UI SF, International Consultation on Incontinence Questionnaire-Urinary Incontinence Short Form; PFMT, pelvic floor muscle training; rTMS: repetitive transcranial magnetic stimulation; SCl, spinal cord injury; UI, urinary incontinence.

these anatomical areas, resulting in significant improvements in controlling voluntary movements. ${ }^{33}$ Based on the principle that high-frequency rTMS $(\geq 5 \mathrm{~Hz})$ increases corticospinal and M1 excitability whereas low-frequency rTMS $(\leq 1 \mathrm{~Hz})$ decreases it, ${ }^{34}$ and considering that recent evidences demonstrated high stimulus intensities could affect activity of spinal cord circuitry. ${ }^{35}{ }^{36}$ We design rTMS protocol according to the trial from Kuppuswamy et al. ${ }^{35}$ Their trial showed this application of $5 \mathrm{~Hz}$ rTMS had been a safe procedure though it did not produce clinical change in chronic, subjects with stable SCI. All participants will use the same rTMS equipment, which uses a figure-of-eight coil with the handle pointing anteromedially over the sensorimotor cortex on one side. The physiotherapist will choose pelvic floor muscle as the reference muscle for applying rTMS, determining the hemisphere and specific part to stimulate. Subjects with anatomical positioning hats will receive rTMS at $5 \mathrm{~Hz}$ for $20 \mathrm{~min}$ per time, five times a week. During a weak (approximately $10 \%)$ voluntary contraction, the equipment will give a stimulation of $80 \%$ of the active motor threshold to elicit a motor evoked potential. Pain or muscle twitch is not elicited by stimulation in the whole process. In addition, all patients will receive standardised PFMT protocol which is as same as the control group. The session of training is scheduled just after the rTMS session.

\section{Group 3 (PFMT with biofeedback and rTMS)}

The subjects in this group will accept a variety of interventions including PFMT, biofeedback and rTMS. The rTMS protocol with identical strength, frequency and duration of stimulation will be applied first, then PFMT protocol with biofeedback will be implemented next.

\section{Interventionist}

All therapists delivering the trial interventions must meet the following requirements: (1) therapists are clinical specialists or qualified physiotherapists who already learn medical knowledge-related SCI; (2) therapists will receive standardised training to ensure standardisation in study process. Standardised training includes lectures on pelvic floor muscle anatomy and UI dysfunction, implementing the PFMT protocol, using the biofeedback equipment and adjusting parameters on associated software, using rTMS unit and mastering anatomy of brain. Each interventionist will be provided the PFMT, biofeedback and rTMS protocol manual which covers details of the intervention parameter and development for all groups.

Although the existing research on rTMS treatment of SCI has not found the occurrence of major adverse reactions, epilepsy, as a known potential risk, should be highly regarded during the course of the study. Qualified therapists should observe subjects at all times and give the appropriate parameters according to the safe limitation of rTMS. All researchers need to have enough skills to deal with unexpected situations and should master the management of seizure and other medical emergencies. Our trial will happen in a hospital where life-support equipment is available. At the same time, there is Data Monitoring Committee (DMC) which helps to complete interim analyses or stop rules. The responsibilities of the DMC are safety monitoring and applying appropriate statistical methods. It will review accumulating data from an ongoing clinical trial and make recommendations which might impact the future conduct of the trial, to ensure that there is no unavoidable increased risk for patients participating in such trials. DMC can provide recommendations for stopping the trial when there are high numbers of protocol violations/deviations or high numbers of patients who withdraw from a study. Considering the psychological factors of patients with SCI, the discomfort of treatment and potential adverse reactions, we design a $20 \%$ dropout rate.

\section{Data collection}

Data will be collected via participant-completed questionnaires at baseline and 1, 3 and 6 months after randomisation. In addition, a pelvic floor muscle exercise diary will be completed in detail by cooperation between the therapist and the patient during the 4-week intervention. After each assessment, the interventionist will record changes in pelvic floor muscle function and bladder symptoms, so that PFMT, biofeedback, and rTMS protocols can be remediation or reinforcement according to the special condition of the patient timely.

The primary outcome is the ICIQ-UI SF, which allows the assessment of the prevalence, frequency, perceived cause of UI and influence on everyday life by questionnaire. The self-reporting questionnaire consists of three 
scored items and an unscored self-diagnostic item, and it is used to diagnose and evaluate the severity of UI. ${ }^{26}$ The total ICIQ-UI SF score ranges from 0 to 21 points with the severity being classified according to the total score: $0-7$ points for mild UI, $8-12$ points for moderate UI and 13-21 points for severe UI. ${ }^{37}$

The secondary outcomes include (1) bladder diary, which is intended to document experiences close to the time that they occur, so it can provide more accurate data about micturition frequency, urinary void volume and urinary symptoms. ${ }^{38}$ The bladder diaries are generally the important tools to evaluate UI symptoms in clinical practice, and the common trial design includes 3-day diaries and 7-day diaries. Considering patients' convenience and compliance, we will use 3-day diaries to obtain details on the participants' bladder function states; (2) pelvic floor muscle function, which is evaluated by perceiving symptoms, physical examination and additional tests such as electromyography, pressure measurements and imaging. ${ }^{39}$ Of them, the physical examination is the most widely used method for testing the pelvic floor muscle function. Before testing, all participants will be required to rest for at least $10 \mathrm{~min}$ to avoid fatigue of pelvic floor muscles, affecting the subsequent evaluation results. During this process, the examiner will teach patients to relax and understand the assessment process. Then doctors and physiotherapists will assess voluntary contraction of the pelvic floor muscles by inspection and palpation; (3) SCI-QoL provides information regarding an individual's satisfaction with their general, physical and emotional health and helps researchers to track changes in those perceptions. The SCI-QoL is a popular instrument for evaluating health-related QoL of adult individuals with traumatic or non-traumatic spinal cord lesions. This questionnaire comprises of three parts, that is, the general quality of life, satisfaction with physical health and satisfaction with psychological health. The total score in each part ranges from 0 to 10 with 0 point indicating completely dissatisfied and 10 points indicating completely satisfied. ${ }^{27}$

Detailed information on the evaluating process is described below to ensure that the participants fill out the questionnaires truthfully. The ICIQ-UI SF scale, bladder diaries and SCI-QoL scale are both subjective and selfreporting questionnaires. All patients should not only understand the meaning of various items in questionnaires but also clear the importance of truthful, timely and complete data entry. Participants will accept the investigator's unified explanation of the questionnaires before filling out the questionnaires, and they will be emphasised to fill them according to their own circumstances during the process of completing the questionnaires. In addition, the patient's daily recorded bladder diaries and ICIQ-UI SF questionnaire have partially repeated questions, thus, the investigator can judge the authenticity of the questionnaire based on the collected bladder diaries. All questionnaires will be completed independently by the subjects. The investigator should not complete the questionnaires on behalf of the subjects or induce the subjects to change their subjective choices through words or other means.

\section{Follow-up}

Once the eligibility of the patients is confirmed and informed consent from the participants is obtained, randomisation will be applied. Then the interventions will be within 24 hours after the randomisation. Participants will undergo 20 treatment sessions, five times a week for 4 weeks. The assessments and visits for patients will be completed by trained therapists in the first month, at 3 months and 6 months after randomisation, with either telephone or in-hospital follow-up. At every participant appointment, trained investigators will collect objective data by physical examination, and participants will complete paper diaries and questionnaires, then experienced staff will collect all data and send it to the computer for data analyses.

\section{Data analysis}

All analyses will be according to the Statistical Analysis Plan and Health Economic Analysis Plan. A single main analysis will be performed at the end of the trial when a 6 -month follow-up has been completed. For processing of variables, means and SDs are used to express continuous measurements and numbers and percentages are for categorical measurements. The primary population for analysis is defined as patients identified according to the intent-to-treat analysis. All participants will be included in the efficacy analyses after randomisation as long as they have a baseline value and at least one measurement. Statistical analyses will be performed using the R software and SPSS software (version 21.0). First, we will determine whether the data are normally distributed used the Shapiro-Wilk test, and Levene's test will be used to examine homogeneity of variance. The mean or mean SD will be used for normally distributed data and median interquartile range (25th-75th percentiles) is for non-normally distributed data. Next, for normally distributed data, we will use Analysis of Variance to evaluate the effectiveness of the intervention, investigating changes of mean in all groups. The significance of differences between the two groups will be evaluated using least significant difference for normally distributed data, moreover, the KruskalWallis test is for non-normally distributed data. Fisher's exact test or $\chi^{2}$ test will be used to compare categorical variables. For all tests, a p value of $<0.05$ will be considered to indicate statistical significance.

\section{Baseline and outcomes analysis}

All baseline measurements will be analysed, including participant's age, body mass index, level of injury and pelvic floor muscle function. For the primary outcome (ICIQ-UI SF score) analysis, a general linear mixed model is used to compare the difference among the control group, the experimental group 1, experimental group 2 and experimental group 3 . The analyses for the 
secondary outcome measurements will be carried out in a similar manner using an appropriate generalised linear model. Descriptive statistics will be presented at each appointment for variable comparison.

It is reported that women over 50 years old usually suffer from laxity or weakness of pelvic floor muscles and atrophy of tissues around the urethra due to decreased oestrogen levels after menopause. ${ }^{40}$ Different scores of ICIQ-UI SF mean different severity of UI and 13 points are the cut-off value of moderate and severe UI. ${ }^{37}$ Considering the potential effect of age and severity of UI and level of injury for outcomes, subgroup analyses will be conducted to determine whether the level of injury, the age of patients $(<50 / \geq 50$ years $)$ and the severity of UI (ICIQ-UI SF score $<13 / \geq 13$ ) at baseline affect the clinical response to treatment.

\section{Sample size}

This article chose the ICIQ-UI SF score as the primary outcome. A study published by Sherburn et al proved that the minimum clinically significant difference was 2.5 for older women with UI, and they estimated the SD was 2.56 conservatively. ${ }^{41}$ Based on results of the related trials, the researchers used 2.52 and 2.56 as the expected absolute intergroup difference and SD. Assuming standardised effect size of 0.25 , a type I error probability of $5 \%$ and a $90 \%$ probability of avoiding a type II error, this trial would require 35 participants in each group at least. Considering $20 \%$ dropout was allowed, finally, approximately 44 patients would be needed in each group.

\section{Ethics and dissemination}

The conduct of the study will conform to the principles of the Declaration of Helsinki and relevant ethical guidelines covering informed consent, confidentiality and data storage. This trial was evaluated by the Clinical Research and Biomedical Ethics Committee of the West China Hospital, Sichuan University and the ethical approval has been obtained (Approval number: 2019-885, date and version identifier: 9 November 2017/V4.0). This trial has been registered in the Chinese Clinical Trial Registry (No ChiCTR1900026126). The Ethics Committee has approved the protocol of study and the informed consent documents. Written and signed informed consent will be obtained from all participants prior to inclusion in the study. The results of the study will be published in scientific journals after peer review and will be shown at related conferences through presentations. The result will be disseminated to most of the population, including participants, researchers, healthcare providers and sponsors.

\section{Confidentiality and monitoring}

Just people related to this trial can access the data and they will take all necessary precautions to maintain confidentiality. All collected paper forms will be stored in a secure manner in the Department of Rehabilitation Medicine Center, West China Hospital, Sichuan University. A locked, secured cabinet will be used to store paper files. For electrical data, it will be stored on a double password-protected computer. Only the primary investigator and the statistician will own the password. The Ethics Committee and Data Monitoring Committee will monitor the trial process.

\section{Patient and public involvement}

The patients and public were not involved in planning and design of this study.

\section{DISCUSSION}

This study will be a randomised controlled trial aiming to investigate the effectiveness and safety of PFMT, biofeedback and rTMS as adjunct treatments for neurogenic bladder dysfunction. It will determine a standardised regimen that consists of PFMT and other conservative treatment protocols for women with neurogenic bladder dysfunction, providing an important reference for the development of a multivariate rehabilitation programme.

As previously mentioned, this is the first randomised trial for combining PFMT and rTMS as therapies for neurogenic bladder dysfunction. PFMT has evolved over decades, as first-line treatment for stress and mixed UI, most works of the literature suggested that it could increase muscle strength and endurance, improving coordination and functional use of bladder. It is a widely recommended conservative treatment combining principles from behavioural science, nursing and muscle physiology. ${ }^{42}$ However, just PFMT is not enough to meet the patients' rehabilitation needs. Applications of rTMS mostly focus on sensory and motor function or neuropathic pain and psychology problems after SCI, while few people know that neuromodulation strategies of rTMS can also be used in bladder dysfunction of incomplete SCI, improving continence by enhancement of residual sensory inputs. In the absence of conservative treatments for UI, if positive results from PFMT and rTMS group are found in this trial, rTMS would be a new behavioural intervention to improve bladder symptoms of women in the future. Biofeedback has become a controversial spot in clinical application because there is no enough evidence to prove the efficacy of biofeedback for PFMT. Thus, the other strength of this study is that the effect of the biofeedback intervention will be evaluated and validated in the clinical protocol with strict quality, and we will collect both subjective and objective data regarding bladder function and QoL using self-reported questionnaires and equipment.

There are some limitations to this trial. First, participant blinding and practitioner blinding cannot be achieved since sham biofeedback and rTMS procedures will not be applied in this trial. All participants will be aware of the intervention methods and personally take part in treatment. In addition, blinding of the assessor may be incomplete because the primary outcome, along with most outcomes of interest, is self-reported. Second, the participants might be heterogeneous due to the broad age range 
of the inclusion criteria, the difference between the types of neurogenic bladder dysfunction and the possibility of comorbidities with other medical conditions. Therefore, when interpreting the study results, consideration should be given to heterogeneity.

In conclusion, this trial will provide preliminary evidence regarding the combined use of PFMT, rTMS and biofeedback. If this study is successful, it will provide scientific evidence to rehabilitation treatment of women with bladder dysfunction after incomplete SCI.

\section{Trial status}

This study is in the process of recruiting participants, and this stage started in January 2020 and will complete in December 2020. The whole trial procedures are expected to complete by the end of June 2021.

\section{Author affiliations}

${ }^{1}$ Department of Rehabilitation Medicine Center, West China Hospital, Sichuan University, Chengdu, Sichuan, PR China

${ }^{2}$ Key Laboratory of Rehabilitation Medicine in Sichuan Province, Chengdu, Sichuan, PR China

${ }^{3}$ State Key Laboratory of Biotherapy, West China Hospital, Sichuan University, Chengdu, Sichuan, PR China

Acknowledgements We would like to show our gratitude to the Department of Rehabilitation Medicine Center in West China Hospital of Sichuan University for their support in facilitating this project. We are also thankful to all the women for participating in the trial.

Contributors $\mathrm{LX}, \mathrm{QW}$ and $\mathrm{CH}$ developed the initial idea of the trial and $\mathrm{QW}$ is the principal investigator. CF, QZ, FX, LP, ZL and LC also participated in the trial design. LX, LP and QW drafted this protocol. All authors commented on drafts of this paper, and read and approved this final manuscript.

Funding This work was supported by the National Natural Science Foundation of China (Grant No 81572231) and the Project of the Science and Technology Department in Sichuan Province (Grant No 2019YJ0119). The funders had no role in study design, data collection and analysis, decision to publish or preparation of the manuscript.

\section{Competing interests None declared.}

Patient and public involvement Patients and/or the public were not involved in the design, or conduct, or reporting or dissemination plans of this research.

Patient consent for publication Not required.

Provenance and peer review Not commissioned; externally peer reviewed.

Open access This is an open access article distributed in accordance with the Creative Commons Attribution Non Commercial (CC BY-NC 4.0) license, which permits others to distribute, remix, adapt, build upon this work non-commercially, and license their derivative works on different terms, provided the original work is properly cited, appropriate credit is given, any changes made indicated, and the use is non-commercial. See: http://creativecommons.org/licenses/by-nc/4.0/.

\section{ORCID iD}

Lin Xu http://orcid.org/0000-0001-7139-6725

\section{REFERENCES}

1 Ruffion A, Castro-Diaz D, Patel H, et al. Systematic review of the epidemiology of urinary incontinence and detrusor overactivity among patients with neurogenic overactive bladder. Neuroepidemiology 2013;41:146-55.

2 Stöhrer M, Goepel M, Kondo A, et al. The standardization of terminology in neurogenic lower urinary tract dysfunction: with suggestions for diagnostic procedures. International continence Society standardization Committee. Neurourol Urodyn 1999;18:139-58.
3 Jeong SJ, Cho SY, Oh S-J. Spinal cord/brain injury and the neurogenic bladder. Urol Clin North Am 2010;37:537-46.

4 Lidal IB, Snekkevik H, Aamodt G, et al. Mortality after spinal cord injury in Norway. J Rehabil Med 2007:39:145-51.

5 Ruffion A, Villar E, Denys P, et al. [Renal failure and neurogenic bladder]. Prog Urol 2007;17:424-30.

6 Lombardi G, Del Popolo G, Macchiarella A, et al. Sexual rehabilitation in women with spinal cord injury: a critical review of the literature. Spinal Cord 2010;48:842-9.

7 Wyndaele J-J. The management of neurogenic lower urinary tract dysfunction after spinal cord injury. Nat Rev Urol 2016;13:705-14.

8 Dumoulin C, Hay-Smith EJC, Mac Habée-Séguin G, et al. Pelvic floor muscle training versus no treatment, or inactive control treatments, for urinary incontinence in women. Cochrane Database Syst Rev 2014;56:300-8.

9 Damen-van Beek Z, Teunissen D, Dekker JH, et al. [Practice guideline 'Urinary incontinence in women' from the Dutch College of General Practitioners]. Ned Tijdschr Geneeskd 2016;160:D674.

10 Rizvi RM, Chughtai NG, Kapadia N. Effects of bladder training and pelvic floor muscle training in female patients with overactive bladder syndrome: a randomized controlled trial. Urol Int 2018;100:420-7.

11 López-Liria R, Varverde-Martínez María de los Ángeles, PadillaGóngora D, et al. Effectiveness of physiotherapy treatment for urinary incontinence in women: a systematic review. J Womens Health 2019;28:490-501.

12 Izak Faiena M, Neal Patel M, Parihar JS, et al. Conservative management of urinary incontinence in women. Prim Care2001;8:153-62.

$13 \mathrm{~B} ø \mathrm{~K}$. Pelvic floor muscle training is effective in treatment of female stress urinary incontinence, but how does it work? Int Urogynecol $J$ Pelvic Floor Dysfunct 2004;15:76-84.

14 Markland AD, Jelovsek JE, Whitehead WE, et al. Improving biofeedback for the treatment of fecal incontinence in women: implementation of a standardized multi-site manometric biofeedback protocol. Neurogastroenterol Motil 2017;29:e12906.

15 Bertotto A, Schvartzman R, Uchôa S, et al. Effect of electromyographic biofeedback as an add-on to pelvic floor muscle exercises on neuromuscular outcomes and quality of life in postmenopausal women with stress urinary incontinence: a randomized controlled trial. Neurourol Urodyn 2017:36:2142-7.

16 Herderschee R, Hay-Smith ECJ, Herbison GP, et al. Feedback or biofeedback to augment pelvic floor muscle training for urinary incontinence in women: shortened version of a Cochrane systematic review. Neurourol Urodyn 2013;32:325-9.

17 Nunes EFC, Sampaio LMM, Biasotto-Gonzalez DA, et al. Biofeedback for pelvic floor muscle training in women with stress urinary incontinence: a systematic review with meta-analysis. Physiotherapy 2019;105:10-23.

18 Chari A, Hentall ID, Papadopoulos MC, et al. Surgical neurostimulation for spinal cord injury. Brain Sci 2017;7:18.

19 Strafella AP, Vanderwerf Y, Sadikot AF. Transcranial magnetic stimulation of the human motor cortex influences the neuronal activity of subthalamic nucleus. Eur J Neurosci 2004;20:2245-9.

20 Lang N, Siebner HR, Ward NS, et al. How does transcranial DC stimulation of the primary motor cortex alter regional neuronal activity in the human brain? Eur J Neurosci 2005;22:495-504.

21 Ellaway PH, Vásquez N, Craggs M. Induction of central nervous system plasticity by repetitive transcranial magnetic stimulation to promote sensorimotor recovery in incomplete spinal cord injury. Front Integr Neurosci 2014;8:42.

22 Joussain C, Denys P. Electrical management of neurogenic lower urinary tract disorders. Ann Phys Rehabil Med 2015;58:245-50.

23 Eric Jelovsek J, Markland AD, Whitehead WE, et al. Controlling anal incontinence in women by performing anal exercises with biofeedback or loperamide (capable) trial: design and methods. Contemp Clin Trials 2015;44:164-74.

24 Chan A-W, Tetzlaff JM, Altman DG, et al. Spirit 2013 statement: defining standard protocol items for clinical trials. Ann Intern Med 2013;158:200-7.

25 Kirshblum SC, Waring W, Biering-Sorensen F, et al. Reference for the 2011 revision of the International standards for neurological classification of spinal cord injury. J Spinal Cord Med 2011;34:547-54.

26 Avery K, Donovan J, Peters TJ, et al. ICIQ: a brief and robust measure for evaluating the symptoms and impact of urinary incontinence. Neurourol Urodyn 2004;23:322-30.

27 Charlifue S, Post MW, Biering-Sørensen F, et al. International spinal cord injury quality of life basic data set. Spinal Cord 2012;50:672-5.

28 Dumoulin C, Hay-Smith J, Frawley H, et al. 2014 consensus statement on improving pelvic floor muscle training adherence: 
international continence Society 2011 State-of-the-Science seminar. Neurourol Urodyn 2015;34:600-5.

29 Vásquez N, Knight SL, Susser J, et al. Pelvic floor muscle training in spinal cord injury and its impact on neurogenic detrusor over-activity and incontinence. Spinal Cord 2015;53:887-9.

30 Laycock J, Jerwood D. Pelvic floor muscle assessment: the perfect scheme. Physiotherapy 2001;87:631-42.

31 Miller JM, Ashton-Miller JA, DeLancey JO. A pelvic muscle precontraction can reduce cough-related urine loss in selected women with mild SUI. J Am Geriatr Soc 1998;46:870-4.

32 Bunday KL, Perez MA. Motor recovery after spinal cord injury enhanced by strengthening corticospinal synaptic transmission. Curr Biol 2012;22:2355-61.

33 Ridding MC, Rothwell JC. Is there a future for therapeutic use of transcranial magnetic stimulation? Nat Rev Neurosci 2007:8:559-67.

34 Ridding MC, Ziemann U. Determinants of the induction of cortical plasticity by non-invasive brain stimulation in healthy subjects. $J$ Physiol 2010;588:2291-304.

35 Kuppuswamy A, Balasubramaniam AV, Maksimovic R, et al. Action of $5 \mathrm{~Hz}$ repetitive transcranial magnetic stimulation on sensory, motor and autonomic function in human spinal cord injury. Clin Neurophysiol 2011;122:2452-61.
36 Bunday KL, Oudega M, Perez MA. Aberrant crossed corticospinal facilitation in muscles distant from a spinal cord injury. PLOS One 2013;8:e76747

37 Karantanis E, Fynes M, Moore KH, et al. Comparison of the ICIQ-SF and 24-hour pad test with other measures for evaluating the severity of urodynamic stress incontinence. Int Urogynecol J Pelvic Floor Dysfunct 2004;15:111-6.

38 Dmochowski RR, Sanders SW, Appell RA, et al. Bladder-health diaries: an assessment of 3-day vs 7-day entries. BJU Int 2005;96:1049-54.

39 Messelink B, Benson T, Berghmans B, et al. Standardization of terminology of pelvic floor muscle function and dysfunction: report from the pelvic floor clinical assessment group of the International continence Society. Neurourol Urodyn 2005;24:374-80.

40 Jundt $\mathrm{K}$, Peschers $\mathrm{U}$, Kentenich $\mathrm{H}$. The investigation and treatment of female pelvic floor dysfunction. Dtsch Arztebl Int 2015;112:564-74.

41 Sherburn M MG. Evaluation of outcome measures for stress urinary incontinence in older women. Neurourol Urodyn 2009;28:715-6.

42 Griebling TL. Re: efficacy of adding behavioural treatment or antimuscarinic drug therapy to $\alpha$-Blocker therapy in men with nocturia. J Urol 2014;192:1495. 